\title{
Characteristics of Beige Adipocytes Induced from White Adipocytes by Kikyo Extract
}

\author{
Naofumi Shiomi, Marie Ito, Keiko Watanabe \\ School of Human Sciences, Kobe College, Nishinomiya, Japan \\ Email:n-shiomi@mail.kobe-c.ac.jp
}

Received 26 May 2016; accepted 18 June 2016; published 21 June 2016

Copyright (C) 2016 by authors and Scientific Research Publishing Inc.

This work is licensed under the Creative Commons Attribution International License (CC BY). http://creativecommons.org/licenses/by/4.0/

(c) (i) Open Access

\begin{abstract}
Beige adipocytes are believed to have a high ability to consume fat. As such, compounds capable of inducing the development of beige adipocytes may be useful as drugs for anti-obesity and antitype 2 diabetes. However, the true nature of beige adipocytes remains unclear. The purpose of this study is to confirm whether or not white adipocyte can differentiate to beige adipocytes and to clarify the characteristics of beige adipocytes. We first searched for an inducer of beige adipocytes and found that kikyo extract, a component of bofu-tsusho-san, was a strong inducer. We then attempted to prove that beige adipocytes could be induced from white adipocytes. Second, we clarified the characteristics of beige adipocytes induced from white adipocytes. The results suggested that beige adipocytes were high-performance adipocytes with a greater ability to synthesize and consume triglyceride and take up glucose than white adipocytes.
\end{abstract}

\section{Keywords}

Beige Adipocyte, UCP1, GLUT4, Diabetes, Obesity, Insulin Resistance

\section{Introduction}

Glucose metabolic disorder and insulin resistance caused by obesity often trigger various diseases, including type 2 diabetes, arteriosclerosis, hyperlipidemia, and ischemic heart disease. Subjects who simultaneously develop these diseases are said to have metabolic syndrome [1]. As the mortality of patients with metabolic syndrome is several times higher than that of normal subjects [2], it is important to prevent glucose metabolic disorders and insulin resistance by reducing body weight to a normal level before the development or at the preliminary stage of metabolic syndrome.

Several studies have suggested that some compounds are effective in reducing weight. For instance, polyphenols can inhibit the absorption of triglycerides [3], and citrate and carnitine inhibit the accumulation of triglycerides by supporting the metabolism [4]-[6]. However, the effects of those compounds are not sufficient in obese 
subjects, as most obese subjects already have a sluggish triglyceride metabolism in addition to an obese constitution. Therefore, the most effective way to reduce the weight of obese subjects is realized by changing their obese constitution to one with a high ability to consume triglycerides.

Uncoupling protein 1 (UCP1), a protein present in the mitochondrial inner membrane, plays a role in nonshivering thermogenesis [7] and has recently attracted attention as a key factor for improving an obese constitution. A study on the effect of UCP1 on an obese constitution using UCP1 transgenic mice found that, when both UCP1 knockout mice and normal mice consumed high-calorie foods for a determined period, the UCP1 knockout mice gained more weight than the normal mice [8]. However, PPAR $\gamma$ knock-in obese mice showed improvement in glucose metabolism disorder and symptoms of diabetes by expressing UCP1 at a high level [9]. In humans, triglycerides are consumed to recover the potential energy lost by UCP1 during nonshivering thermogenesis [10] [11]. Therefore, enhancement of the UCP1 activity is expected to effectively improve an obese constitution and symptoms of type 2 diabetes [12].

Given that UCP1 is expressed only in brown adipocyte tissue (BAT) [13] [14], an increase in BAT is the best way to increase UCP1 expression. Brown adipocytes are long believed to be unable to be differentiated from myoblasts or white adipocytes, and the amount cannot be controlled. However, recent studies have suggested that myoblasts can transform to brown adipocyte by expressing PRDM16 at high levels [15] [16], and white adipocytes may also be able to differentiate into brown adipocytes via exercise or chilly stimulation for a long period of time [17] [18]. As it is given that the brown adipocytes that develop from white adipocytes differ from the natural brown adipocytes present in the body based on their expression of the Myf5 gene, these induced to brown adipocytes are called "beige adipocytes (or brite adipocytes)" to distinguish them from natural ones.

Triglycerides that accumulate in white adipocyte tissue are first degraded to fatty acid and glyceride in white adipocytes, then transferred to muscle cells via the circulation, and finally consumed as energy in muscles. If white adipocytes accumulating fat can be changed to beige adipocyte, they can directly consume the triglycerides themselves at a high rate, without transport to muscle. Compounds capable of transforming white adipocytes to beige adipocyte will therefore be extremely useful in improving an obese constitution. However, few compounds which can efficiently promote such differentiation have been discovered.

The purpose of this study was to identify such compounds and clarify the characteristics of beige adipocytes induced from white adipocytes. We therefore focused on Chinese herbal medicines and identified kikyo (Platycoi radix) extract as a strong inducer of differentiation. We also examined the characteristics of the beige adipocytes induced by kikyo extract and found that these adipocytes were high-performance cells with high triglyceride consumption and glucose metabolism.

\section{Materials and Methods}

\subsection{Extract Solution of Chinese Herbal Medicine}

Bofu-tsusho-san, a mixture of Chinese herbal medicines (crude drugs), contains sixteen kinds of crude drugs. Thirteen of these drugs-mao (Ephera herba), bofu (Saposhnikovae radix), keigai (Schizonepetae herba), daio (Rhei rhizoma), kanzo (Glycyrhizae raix), rengyo (Forsythiae fructus), kikyo (Platycoi radix), senkyu (Chuanxiong rhizoma), ogon (Scutellariae radix), sanshinshi (Gardeniae fructus), toki (Angelicae raix), shyakuyaku (Paeniae radix), hakka (Menthae haplocalysis herba), and bakujutsu (Atractyloidis macrocephalae rhizo$m a$ ) - were purchased from a drug store and used in the preliminary test for the screening of effective components. The remaining three components—bosho (Natrium sulfrus), sekko (Gypsum fibrosum), and kasseki (Talcum crystallinum) - were not used, as their main compound was not a crude extract but a chemical compound. Each crude drug $(100 \mathrm{mg})$ and $1 \mathrm{~mL}$ water was mixed in a 2.0-mL micro-tube, and the effective compounds were extracted by sterilization for $1 \mathrm{~min}$ at $120^{\circ} \mathrm{C}$. The solution was used as extract solution.

\subsection{Cell Line, Medium and Additives}

A murine cell line of 3T3-L24 white adipocytes, which had been obtained by differentiation from 3T3-L1 preadipocytes at our laboratories [6], was used for this study. Dulbecco's modified Eagle's medium containing sodium bicarbonate (MP Biomedicals Inc., IIIkirch, France) and penicillin/streptomycin solution (250 U/mL and $250 \mu \mathrm{L} / \mathrm{mL}$ ) were mixed with fetal bovine serum (FBS) at a ratio of 9:1. The mixture (DMEM) was used as a culture medium. Glucose-free DMEM (Thermo Fisher Scientific Inc., Waltham, MA, USA) without FBS was 
also used for the consumption test for triglycerides and the uptake test for glucose. Insulin, noradrenaline, and oleic acid were dissolved in PBS, $1 \mathrm{mM} \mathrm{HCl}$ solution, and ethanol at final concentrations of $1 \mathrm{mg} / \mathrm{mL}, 20 \mathrm{mM}$, and $400 \mathrm{mM}$, respectively. 3T3-L24 white adipocytes were cultured in DMEM containing $3.3 \mu \mathrm{L} / \mathrm{mL}$ of kikyo extract solution for 3 or 14 days, and the obtained cells were named 3T3-BA1. DMEM containing $3.3 \mu \mathrm{L} / \mathrm{mL}$ of kikyo extract solution was used as a basic medium for the culture of 3T3-BA1 cells.

\subsection{Gene Expression}

3T3-L24 adipocytes ( $2 \times 10^{4}$ cells) were pre-cultured in 25- $\mathrm{cm}^{2}$ culture flasks containing $6 \mathrm{~mL}$ of DMEM for 16 $\mathrm{h}$ to promote cell attachment. The medium was changed to $6 \mathrm{~mL}$ of DMEM, DMEM containing $20 \mu \mathrm{L}$ of extract solution of crude drugs, and DMEM containing $10 \mu \mathrm{M}$ noradrenaline for 3 days to induce gene expression.

The total mRNA in the cells was purified, and cDNA was synthesized using an RNeasy Lipid Tissue Mini kit and a QyantiTeck Reverse Transcription Kit (Qiagen K. K., Tokyo, Japan). The gene expression was examined using a real-time polymerase chain reaction (PCR). The reaction mixture was prepared with a Rotor-Gene SYBR Green PCR Kit. The primers (QuantiTeck Primer Assays) obtained from Qiagen K. K. were used for the detection of $\beta$-actin, COX8b, CDK5, CIDEAR, PRDM16, UCP1, PPAR $\alpha$, PPAR $\gamma, \mathrm{CEBP} / \alpha, \mathrm{CEBP} / \beta, \mathrm{FABP} 4$, leptin, resistin, adiponectin, TNF $\alpha$, ADR3, and IR2 genes. Real-time PCR was performed using the Rotor-Gene TM device (Qiagen K. K.), and the reaction was carried out for 50 - 80 cycles of treatment at $95^{\circ} \mathrm{C}$ for 5 seconds and $65^{\circ} \mathrm{C}$ for 30 seconds. The $\mathrm{Ct}$ value (threshold line 0.1 ) was determined using $\beta$-actin as a standard gene. Three independent culture experiments were performed, and the average values, standard deviations (SDs) and p-values were calculated.

\subsection{Apparent Color of Cells and Staining of Oil Droplets}

The apparent color of 3T3-L24 cells and 3T3-BA1 cells was observed. The 3T3-L24 cells and 3T3-BA1 cells (5000 cells each) were cultured in a 24-well culture plate containing $1 \mathrm{~mL}$ of DMEM alone and DMEM + kikyo extract $(3.3 \mu \mathrm{L} / \mathrm{mL}$ medium), respectively, for 3 days. The wells were washed with PBS, and the apparent color of cells was observed with a microscope.

Oil drops accumulated in the cells were stained using the oil red method. The 3T3-L24 cells and 3T3-BA1 cells (5000 cells each) were cultured in a chamber slide containing $0.2 \mathrm{mM}$ of oleic acid and $1 \mathrm{~mL}$ of either the DMEM alone or the DMEM $+3.3 \mu \mathrm{L}$ of kikyo extract, respectively, for 2 days. After washing the wells twice with $1 \mathrm{~mL}$ PBS, the cells cultured in the chamber slide were incubated with $10 \%$ formalin solution for 10 min to fix the cells, and isopropanol solution (60\%) was added and incubated for $1 \mathrm{~min}$. The cells were then washed 3 times with $2 \mathrm{~mL}$ of PBS and stained red with $250 \mu \mathrm{L}$ Oil red O solution (60\%) for 15 min. The cells were washed once with $60 \%$ isopropanol solution and twice with $2 \mathrm{~mL}$ of PBS. The oil droplets stained red were observed with a microscope.

\subsection{Assays of UCP1 and GLUT4 with a Fluorescence Microscope and a Flow Cytometer}

Assays with a fluorescence microscope were performed by the following procedure: 3T3-L24 cells and 3T3BA1 cells were cultured on the cover glass (13 mm in diameter, Matunami Glass Ind. Ltd., Osaka, Japan) which was placed in a 24 well-plate containing $1 \mathrm{~mL}$ of DMEM alone and DMEM $+3.3 \mu \mathrm{L}$ of kikyo extract, respectively, for 3 days. The cells were pretreated by the following procedure: The cells cultured on the cover glass were fixed using phosphate buffer solution containing 4\% formaldehyde (WAKO Chemical Co. Ltd., Kyoto, Japan) for 10 min, washed twice with PBS solution, incubated with PBS solution containing 0.5\% TritonX-100 for $5 \mathrm{~min}$, and washed 3 times with PBS. Blocking treatment was performed with PBS containing 1\% bovine serum albumin (BSA) for $30 \mathrm{~min}$, and the cells were washed twice with PBS solution.

The amount of UCP1 and GLUT4 were determined by diluting rabbit anti-GLUT4 polyclonal antibody solution (G4048; Sigma-Aldrich) or anti-UCP1 polyclonal antibody solution (bs-192R; Biosis Co.) to 1/200 volume with PBS $(100 \mu \mathrm{L})$, and the diluted antibody solution was incubated with the pretreated cells for $1 \mathrm{~h}$ and washed 3 times with PBS. Then, FITC-conjugated or Cy3-conjugated anti-rabbit IgG goat polyclonal antibody (secondary antibody) was diluted to 1/400 volume with PBS, and the secondary antibody solution was incubated for 30 min and washed 3 times with PBS. Finally, the chromosomal DNAs of the cells was stained with Hoechst 33342 solution for $10 \mathrm{~min}$ as a control of fluorescence intensity and washed 3 time with PBS. The fluorescence was 
observed with a fluorescence microscope (EVOS ${ }^{\circledR}$ FL Auto Cell Imaging System; Thermo Fisher Scientific Inc.).

A flow cytometer analysis was performed by the following procedure: 3T3-L24 cells and 3T3-BA1 cells were cultured in 25- $\mathrm{cm}^{2}$ flasks containing $6 \mathrm{~mL}$ of DMEM and DMEM $+3.3 \mu \mathrm{L}$ of kikyo extract, respectively, for 3 days. UCP1 or GLUT4 was combined with anti-UCP1 and anti-GLUT4 antibodies and FITC-conjugated secondary antibody via a procedure similar to that described above. The cells were peeled with $0.25 \%$ trypsin solution and suspended in PBS. The mean values of the fluorescence intensity in 5000 cells were determined using a FACSCaliburTM HD flow cytometer (BD Biosciences, Tokyo, Japan).

\subsection{Number of Mitochondria and the Harmful Effects of Oleic Acid on Mitochondria}

The amount of mitochondria was determined using a Molt View Green (Funakoshi Co. Ltd., Tokyo, Japan). 3T3-L24 and 3T3-BA1 cells $\left(2 \times 10^{5}\right.$ cells) cultured in $25 \mathrm{~cm}^{2}$-culture flask had their media changed to $6 \mathrm{~mL}$ of DMEM containing $50 \mathrm{nM}$ of Molt View Green and were incubated for $30 \mathrm{~min}$. The cells were suspended in 1 $\mathrm{mL}$ of PBS followed by peeling with $0.25 \%$ trypsin and washed with PBS. The cells $\left(1 \times 10^{6}\right.$ cells) were suspended in $150 \mu \mathrm{L}$ of PBS, and the cell suspension was placed in the wells of a 96-well plates. The cells were disrupted using an ultrasonic disrupter (TOMY SEIKO Co. Ltd., Tokyo, Japan), and their fluorescence (excitation/emission: 485/535 nm) was measured using a fluorescent plate reader. A total of four independent experiments were performed.

The harmful effects of oleic acid on mitochondrial activity were examined using a Cell Counting Kit-8 (DOJINDO Laboratories, Kumamoto, Japan). Solutions of 40, 80 and $120 \mathrm{mM}$ oleic acid were prepared by diluting $400 \mathrm{mM}$ oleic acid with ethanol. The 3T3-L24 cells and 3T3-BA1 cells (5000 cells/well) were cultured in a 96-well culture plate containing $200 \mu \mathrm{L}$ of DMEM and DMEM $+3.3 \mu \mathrm{L}$ of kikyo extract, respectively, for 16 h. Then, all of the media were changed to $200 \mu \mathrm{L}$ of DMEM containing $2 \mu \mathrm{L}$ of various concentrations of oleic acid solution and incubated for $2 \mathrm{~h}$. After the wells were washed twice, all of the media were changed to $100 \mu \mathrm{L}$ of fresh DMEM containing $5 \mu \mathrm{L}$ of the Cell Counting Kit-8 solution and incubated for $4 \mathrm{~h}$. The absorbance of the culture broth at $450 \mathrm{~nm}$ was measured. A total of four independent experiments were performed. The average values, SDs, and p-values were calculated.

\subsection{Rates of Triglyceride Synthesis and Consumption}

3T3-L24 cells and 3T3-BA1 cells $\left(2 \times 10^{5}\right.$ cells) were cultured in 3 wells each of a 6 -well pate containing $2 \mathrm{~mL}$ of DMEM for $6 \mathrm{~h}$ to promote cell attachment and then for another $16 \mathrm{~h}$ after $2 \mu \mathrm{L}$ of oleic acid solution $(0.4 \mathrm{mM}$ at the final concentration) was added to the medium. Two similar 6-well plates were prepared. One plate was used for the measurement of triglyceride synthesis, and the medium of the other plate was washed 3 times with PBS, $1 \mathrm{~mL}$ of glucose-free DMEM (without FBS) or glucose-free DMEM $+20 \mu \mathrm{M}$ of noradrenaline was added, and the plates were incubated for another $16 \mathrm{~h}$. This plate was used for the measurement of triglyceride consumption.

The concentration of triglyceride was determined by the previously described methods [6], as follows: The cells were peeled using $0.25 \%$ of trypsin and suspended in $2 \mathrm{~mL}$ of DMEM. The total number of cells was counted using a cell counter (WAKENYAKU CO. LTD., Kyoto, Japan). After the cells were harvested, they were suspended with $0.1 \mathrm{~mL}$ of distilled water and disrupted using an ultrasonic disrupter for $30 \mathrm{sec}$. Triglyceride was extracted using $0.3 \mathrm{~mL}$ of diethylether and dried at room temperature. The concentration of triglyceride was determined using a Triglyceride E Test Wako (Wako Chemical Co. Ltd., Osaka, Japan). Three independent culture experiments were performed, and the average values, SDs and p-values were calculated.

\subsection{Rate of Glucose Uptake}

The rate of glucose uptake into cells was determined using a Glucose Uptake Cell-Based Assay Kit (Carman Chemical, Ann Arbor, MI, USA). 3T3-L24 cells and 3T3-BA1 cells (5000 cells) were cultured in 8 wells each of a 96-well white plate containing $100 \mu \mathrm{L}$ of DMEM per well for $16 \mathrm{~h}$. All of the media were changed to DMEM and DMEM $+1 \mu \mathrm{g} / \mathrm{mL}$ insulin in every 4 wells and incubated for $1 \mathrm{~h}$, and were washed once with PBS. All of the media were then changed to $100 \mu \mathrm{L}$ of glucose-free medium containing $10 \mu \mathrm{g} / \mathrm{mL}$ of 2-deoxy-2-[(7nitro-2,1,3-benzoxadiazol-4-yl)amino]-D-glucose (2-NBDG), the fluorescent glucose analog, and incubated for 
10 min to allow for uptake. The amount of 2-NBDG was measured using a fluorescence plate reader (excitation/emission: 485/535 nm) after the cells were washed with the wash solution. The cells were collected by peeling with $0.25 \%$ of trypsin solution, and the total cell number was counted using a cell counter. The average values, SDs, and p-values were calculated from a total of four experiments.

\section{Results}

\subsection{Screening for the Most Effective Crude Drug in Inducing Beige Adipocytes}

Compounds which can effectively change white adipocytes to brown adipocytes are expected to be useful for improving an obese constitution. While bofu-tsusho-san has been used as an anti-obesity drug in Japan, its ability to induce the development of brown adipocyte is unknown. We therefore examined whether or not any of the 13 crude components of bofu-tsusho-san had an ability to induce the development of brown adipocytes.

In preliminary experiments performed to determine a candidate, 3T3-L24 white adipocytes $\left(5 \times 10^{4}\right.$ cells) were cultured in DMEM solutions containing 13 different extract solutions of the crude drugs for 3 days. The expression of uncoupling protein 1 (UCP1) and PRD1-BF1-RIZ1 homologous domain containing 16 (PRDM16) was then determined via real-time PCR. PRDM16 plays a role in the differentiation of white adipocytes to brown adipocytes [15] [16], and UCP1 is expressed only in brown adipocytes. These proteins are therefore the most important marker genes for distinguishing white and brown adipocytes.

The results of preliminary experiments suggested that the extract solutions of keigai, daio, kikyo, senkyu, sanshinshi, and toki induced the expression of PRDM16 and UCP1 genes at high levels (data not shown). We ultimately chose to use kikyo extract in subsequent experiments, as this compound was the most effective in enhancing the PRDM16 gene. The cells whose differentiation was induced by the kikyo extract were named "3T3-BA1" cells. In preliminary experiments with these 3T3-BA1 cells, gene expression was most clear after three-day stimulation by kikyo extract, but stimulation of at least seven days was necessary to obtain stable induced cells. Therefore, cells cultured for 3 and 14 days were respectively used for the tests of gene expression and characteristics of cells. These cells were denoted as "3T3-BA1 (3-day stimulation)" and "3T3-BA1 (14-day stimulation)” when necessary.

Further investigations were performed to confirm the inductive activity of kikyo extract. Figure 1(a) shows the ratios of gene expression observed in the 3T3-BA1 cells (3-day stimulation) to those observed in 3T3-L24 adipocytes. Cytochrome c oxidase subunit VIII (COX8), cyclin-dependent kinase 5 (CDK5), and cell deathinducing DFFA-like effector a (CIDEA) were added as marker genes of brown adipocytes. These proteins are related to mitochondria and are suggested to be strongly expressed in brown adipocytes [19] [20]. The average expression of COX8b, CDK5, CIDEA, PRDM16, and UCP1 genes observed in the 3T3-BA1 cells was 10.0, 1.4, 2.8, 2.2, and 3.5 times higher than those in those observed in 3T3-L24 adipocytes, respectively. The result suggest that kikyo extract contains an inducer of brown adipocytes.

In addition, the inductive activity of kikyo extract was compared with noradrenaline, as noradrenaline has the ability to differentiate white adipocytes to beige ones [21]. Figure 1(b) shows the ratios of expression in the cells induced by noradrenaline to that of 3T3-L24 cells when the 3T3-L24 cells were cultured in DMEM containing $10 \mu \mathrm{M}$ noradrenaline for 3 days. The expression of COX8, CDK5, CIDEA, PRDM16, and UCP1 genes observed in the cells induced by noradrenaline were 2.2, 1.7, 1.9, 2.0, and 1.6 times higher than those observed in 3T3-L24 adipocytes, respectively, and these values were lower than those of 3T3-BA1 cells. These results suggest that the inductive activity of kikyo extract is sufficiently strong.

\subsection{Confirmation That 3T3-BA1 Cells Are Beige Adipocytes}

To confirm whether or not the 3T3-BA1 cells were indeed beige adipocytes, several characteristics were examined. First, the apparent color and number of mitochondria in the 3T3-BA1 cells (14-day stimulation) were compared with those of 3T3-L24 white adipocytes. Figure 2(a) shows the apparent colors of the cells cultured in DMEM; the 3T3-BA1 cells were slightly browner than the 3T3-L24 adipocytes. Figure 2(b) shows the number of mitochondria determined using Molt View Green (a fluorescent dye). As the fluorescent dye selectively combines with mitochondria, the fluorescent intensity is roughly proportional to the number of mitochondria. As shown in Figure 2(b), there were 1.5 times more mitochondria in the 3T3-BA1 cells than in the 3T3-L24 adipocytes. Given that natural brown adipocytes get their color from their high numbers of mitochondria containing 


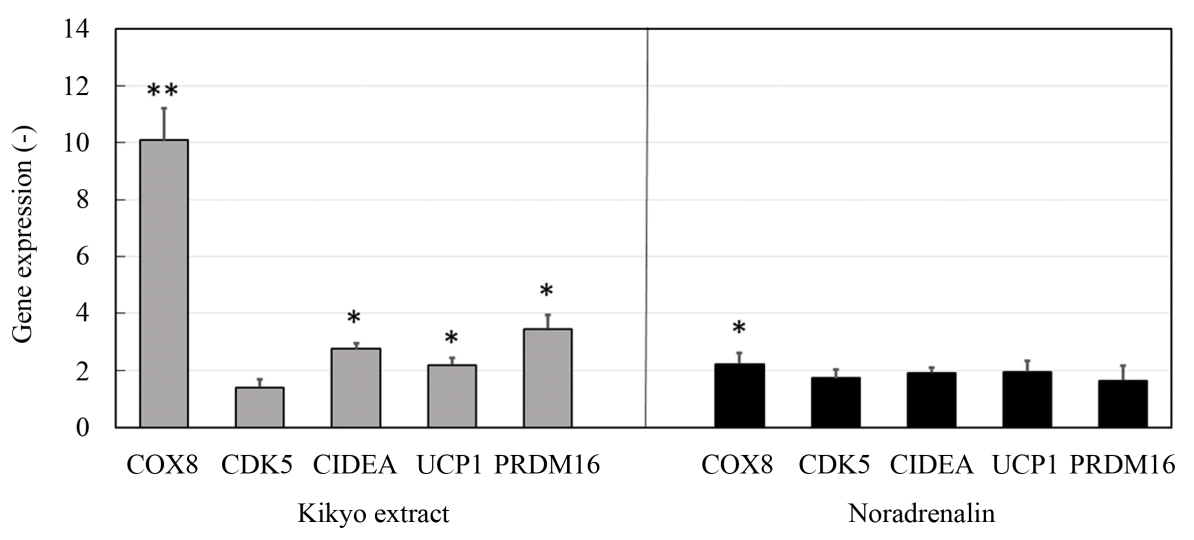

(a)

(b)

Figure 1. The inductive activities of kikyo extract and noradrenaline. (a): Ratios of mRNA levels observed in the cells induced by kikyo extract to those observed in the 3T3-L24 cells. (b) Ratios of mRNA levels observed in the cells induced by noradrenaline to those observed in the 3T3-L24 cells. Bar: mean \pm SD. ${ }^{* *} \mathrm{p}<0.01$ and ${ }^{*} \mathrm{p}<0.05$ vs. 3T3-L24 $(\mathrm{n}=3)$.

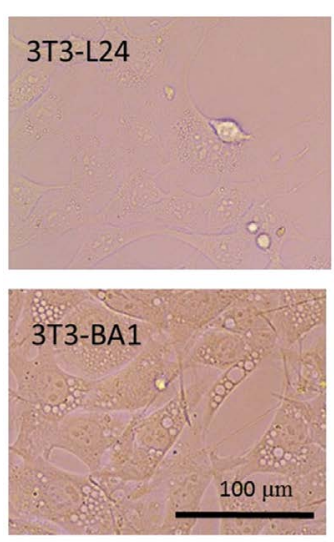

(a)

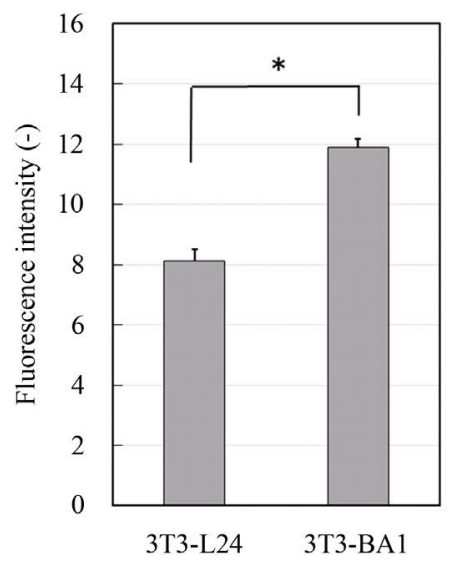

(b)

Figure 2. The apparent color and number of mitochondria in 3T3-BA1 cells. (a) Photographs of 3T3-L24 and 3T3-BA1 cells. (b) Fluorescent intensity of the 3T3-L24 and 3T3-BA1 cells stained with Molt View Green. Bar: mean \pm SD. ${ }^{*} \mathrm{p}<0.05$ vs. 3T3-L24 $(n=4)$.

brown ferric ion, the apparent nature of 3T3-BA1 cells closely resembles the natural brown adipocytes [22].

The expression of UCP1 in 3T3-BA1 cells was also confirmed. Anti-UCP1 antibody which combined with Cys3-conjugated secondary antibody was combined with the UCP1 protein contained in 3T3-L24 and 3T3-BA1 cells. UCP1 appears red under a fluorescence microscope. Line (a) of Figure 3(a) shows the expression of UCP1 in the cells. As shown in the two photos of line (a), UCP1 was not observed in 3T3-L24 adipocytes, but a large amount of UCP1 was observed in 3T3-BA1 cells. Additionally, the average fluorescence intensity of UCP1 was determined using a flow cytometer (FACS). Anti-UCP1 antibody which combined with FITC-conjugated secondary antibody was combined with UCP1, and the fluorescence intensity of 5,000 cells was determined (Figure 3(b)). As shown in Figure 3(b), the average fluorescence intensity of UCP1 per 3T3-BA1 cell was 1.7 times that per 3T3-L24 adipocyte. These results suggest that the 3T3-BA1 cells show a similar nature to brown adipocytes, since UCP1 protein is only expressed in brown adipocytes [22]. On comprehensive consideration of these findings, we determined that the 3T3-BA1 cells were "beige adipocytes" (brown adipocytes induced from white adipocyte).

\subsection{Triglyceride Synthesis by 3T3-BA1 Beige Adipocytes}

The ability of 3T3-BA1 beige adipocytes to synthesize triglycerides was examined. 3T3-BA1 (14-day stimulation) 


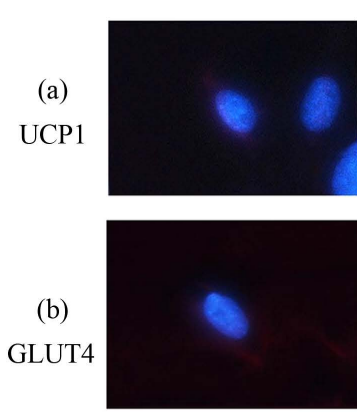

3T3-L24
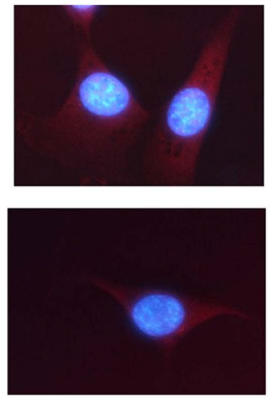

3T3-BA1

(a)

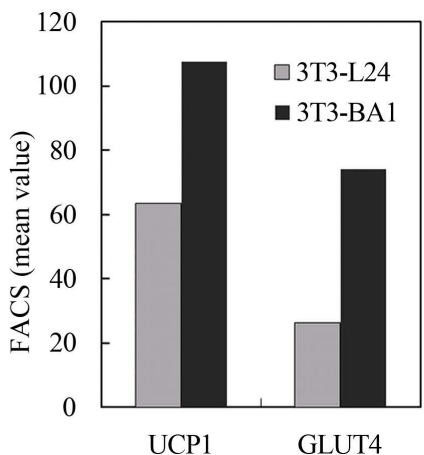

(b)

Figure 3. Expression of UCP1 and GLUT4 in 3T3-BA1 cells. (a) Photographs of UCP1 and GLUT4 in 3T3-L24 and 3T3-BA1 cells. UCP1 and GLUT4 were stained by anti-UCP1 and anti-GLUT4 antibody combining with Cy3-conjugated secondary antibody, and the chromosomal DNA was stained with Hoechst 33342. (b) Mean value of fluorescence intensity per cell in the FACS analysis. UCP1 and GLUT4 contained in 3T3-L24 and 3T3-BA1 cells were stained by anti-UCP1 and anti-GLUT4 antibody combining with FITC-conjugated secondary antibody. Gray bar: 3T3-L24, black bar: 3T3-BA1.

and 3T3-L24 cells were cultured in DMEM containing $0.2 \mathrm{mM}$ oleic acid for 2 days and then stained using the oil red method. Figure 4(a) shows the photographs of the oil droplets from those cells. The 3T3-BA1 cells were able to accumulate many oil droplets, similar to 3T3-L24 adipocytes. These results suggest that the 3T3-BA1 beige adipocytes retained the ability of white adipocytes to synthesize triglycerides.

We next compared the gene expression between the 3T3-BA1 and 3T3-L24 adipocytes. Six genes-peroxisome proliferator-activated receptor $\alpha(\mathrm{PPAR} \alpha)$, PPAR $\gamma$, CCAAT-enhancer-binding proteins $\alpha$ (C/EBP $\alpha)$, C/ $\mathrm{EBP} \beta$, fatty acid binding protein 4 (FABP4) and coactivator- $1 \alpha$ (PGC1 $\alpha$ ) genes - were selected as target genes, as they are transcriptional factors of white adipocytes and play a role in inducing triglyceride synthesis [15] [23]. Figure 4(b) shows the ratios of the gene expression observed in the 3T3-BA1 cells (3-day stimulation) to that observed in the 3T3-L24 cells. The average levels of PPAR $\alpha, \operatorname{PPAR} \gamma, \mathrm{C} / \mathrm{EBP} \alpha, \mathrm{C} / \mathrm{EBP} \beta, \mathrm{FABP} 4$, and PGC1 $\alpha$ genes observed in the 3T3-BA1 cells were 1.8, 1.4, 3.0, 2.0, 2.5 and 1.7 times those observed in the 3T3-L24 adipocytes. The result suggests that the 3T3-BA1 beige adipocytes have a greater ability to synthesize triglyceride than the 3T3-L24 cells.

To confirm this finding, the synthesis rate of triglycerides in the 3T3-BA1 cells was compared with that of the 3T3-L24 cells. The 3T3-BA1 cells (14-day stimulation) and 3T3-L24 cells were cultured in the DMEM containing $0.4 \mathrm{mM}$ oleic acid over a short duration $(16 \mathrm{~h})$, and the amount of triglycerides generated was determined. The gray bars of Figure 5(a) show the results. The average amount of triglycerides synthesized by 3T3-BA1 cells in $16 \mathrm{~h}$ of culture was 2.8 times that by 3T3-L24 white adipocytes.

\subsection{Triglyceride Consumption by 3T3-BA1 Beige Adipocytes}

The triglyceride consumption by 3T3-BA1 beige adipocytes was examined. White adipocytes accumulating many oil droplets generally show a reduced consumption of triglycerides, due to the dysfunction induced by fatty acids [24]. We therefore first examined the harmful effects of fatty acid on the mitochondrial activity. The 3T3-L24 and 3T3-BA1 cells (14-day stimulation) were cultured in DMEM containing 0.4, 0.8, and $1.2 \mathrm{mM}$ of oleic acid for $2 \mathrm{~h}$ to replicate damage by fatty acid. Figure 5(b) shows the ratios of mitochondrial activity in these cells to that in control cells (cells cultured in medium without oleic acid). When the 3T3-L24 cells were cultured in the medium containing 0.8 and $1.2 \mathrm{mM}$ oleic acid, the mitochondrial activities fell to $55 \%$ and $31 \%$ due to the harmful effects of fatty acid. In contrast, the decrease in the activities in 3T3-BA1 beige adipocytes were only $4 \%$ and $18 \%$ following culture in medium containing 0.8 and $1.2 \mathrm{mM}$ oleic acid. These results suggested that the triglyceride consumption in 3T3-BA1 cells accumulating triglyceride could be maintained at a high level without inducing any harmful effect due to fatty acid.

To confirm this finding, the rate of triglyceride consumption in 3T3-BA1 cells was compared with that in 3T3-L24 cells under triglyceride-accumulated conditions. As already shown in the gray bars in Figure 5(a), 

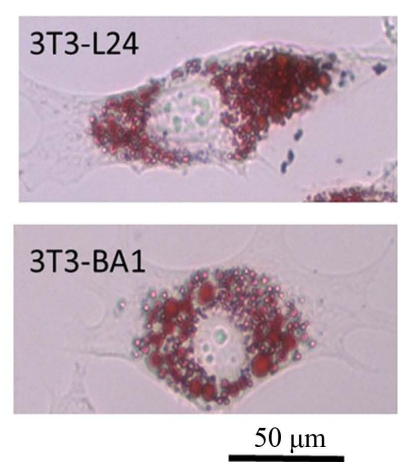

(a)

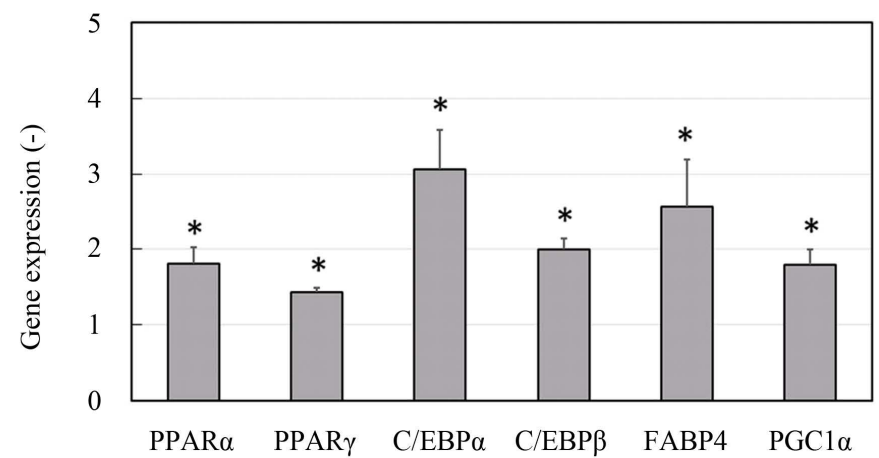

(b)

Figure 4. The accumulation of oil and the gene expression in 3T3-BA1 cells. (a) Photographs of oil droplets stained red in 3T3-L24 and 3T3-BA1 cells. (b) Ratios of the expression of the transcriptional genes related to white adipocytes observed in 3T3-BA1 cells to those observed in 3T3-L24 cells. Bar: mean \pm SD. ${ }^{*} \mathrm{p}<0.05$ vs. 3T3-L24 $(\mathrm{n}=3)$.

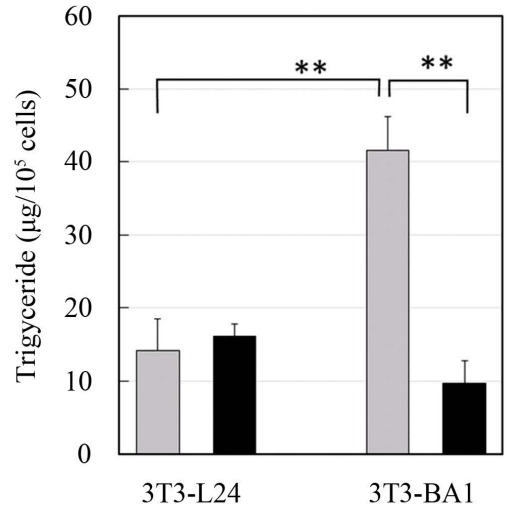

(a)

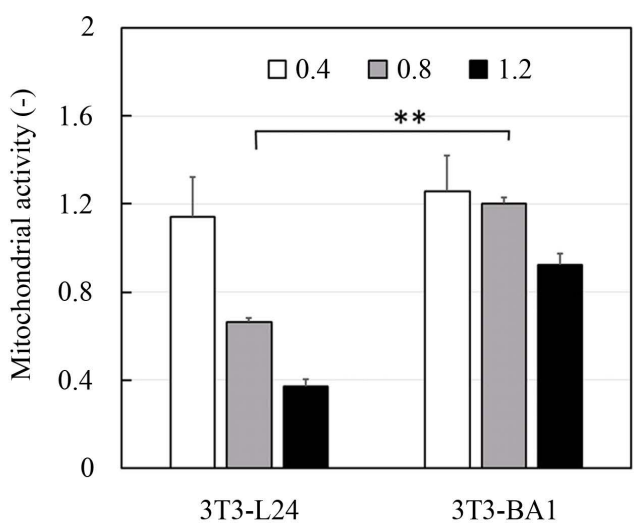

(b)

Figure 5. Triglyceride metabolism and harmful effect of oleic acid in 3T3-BA1 cells. (a) The rates of triglyceride synthesis and consumption in 3T3-BA1 cells. Gray bars: The amounts of triglyceride synthesized in 3T3-L24 and 3T3-BA1 cells cultured in DMEM containing either $0.4 \mathrm{mM}$ oleic acid alone or $0.4 \mathrm{mM}$ oleic acid and $3.3 \mu \mathrm{L} / \mathrm{ml}$ of extract solution of kikyo for $16 \mathrm{~h}$. Black bars: Amounts of triglyceride in 3T3-L24 and 3T3-BA1 cells cultured in glucose-free DMEM for $16 \mathrm{~h}$ after triglyceride accumulation. (b) Mitochondrial activity of the 3T3-L24 and 3T3-BA1 cells cultured in DMEM containing $0.4,0.8$, and $1.2 \mathrm{mM}$ oleic acid. The vertical axis indicates the ratio of the activity of the cells cultured in the medium containing oleic acid to that of control cells (the cells cultured DMEM without oleic acid) Bar: mean \pm SD. ${ }^{* *} \mathrm{p}<0.01$ and ${ }^{*} \mathrm{p}<0.05$ vs. control $(\mathrm{n}=4)$.

triglycerides were accumulated in the cells cultured in DMEM containing $0.4 \mathrm{mM}$ oleic acid for $16 \mathrm{~h}$. The cells accumulating triglyceride were subsequently cultured in glucose-free DMEM without oleic acid for another $16 \mathrm{~h}$ to induce the consumption of triglycerides. The black bars in Figure 5(a) show the residual triglyceride in the 3T3-L24 and 3T3-BA1 cells after this consumption of triglycerides. The amount of triglyceride in 3T3-BA1 cells decreased from $42 \mu \mathrm{g}$ to $8 \mu \mathrm{g}$, but the value in 3T3-L24 adipocytes did not decrease. These results suggest that the rate of triglyceride consumption as well as synthesis in 3T3-BA1 beige adipocytes is much higher than that in 3T3-L24 white adipocytes.

\subsection{Uptake Rate of Glucose in 3T3-BA1 Beige Adipocytes}

We next examined the rate of uptake of glucose to clarify the positive effects on insulin resistance. The expression of adipocytokines was examined first, as adipocytokines are hormones that control the uptake rate of glucose [1]. The gray bars of Figure 6(a) show the ratios of the expression of genes observed in 3T3-BA1 cells (3-day stimulation) to those in 3T3-L24 cells. The expression of leptin and TNF $\alpha$ in 3T3-BA1 cells was 3.2 and 


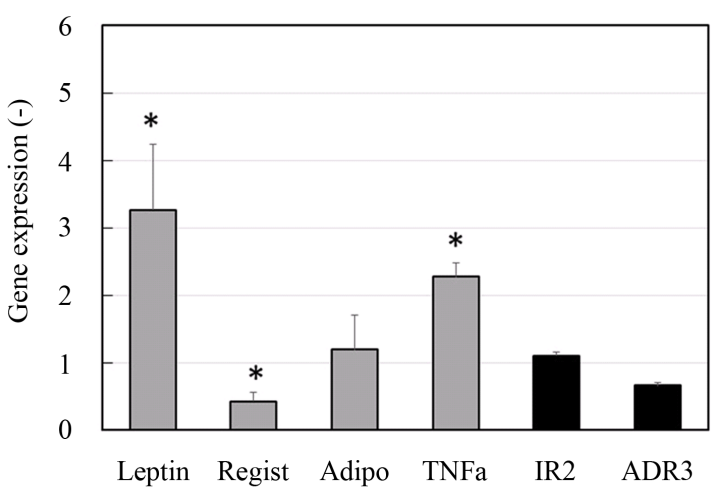

(a)

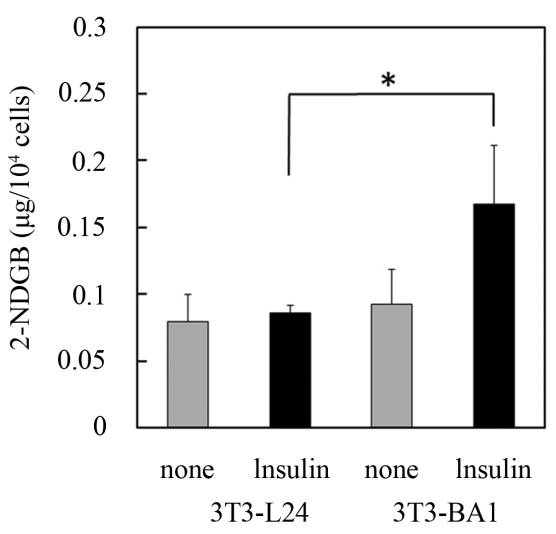

(b)

Figure 6. Expression of adipocytokines and uptake rate of glucose in 3T3-BA1 cells. (a) The ratios of the mRNA levels of the adipocytokines observed in the 3T3-BA1 cells to those observed in the 3T3L24 cells. Resist and Adopo at the horizontal axis indicate resistin and adiponectin. B: The uptake amount of glucose in 3T3-L24 and 3T3-BA1 cells (104 cells). 3T3-L24 and 3T3-BA1 cells were incubated in glucose-free DMEM containing 2-NDGB (none) or $1 \mu \mathrm{g} / \mathrm{mL}$ of insulin (Insulin) for 20 min. Bar: mean \pm SD. ${ }^{*} \mathrm{p}<0.05$ vs. 3T3-L24 $(\mathrm{n}=3)$.

2.2 times that in 3T3-L24 cells, but those of adiponectin and resistin were roughly the same and 60\% lower, respectively. Leptin promotes glucose metabolism, and resistin inhibits it by inhibiting the insulin signal [25] [26]. Therefore, glucose metabolic disorders (or insulin resistance) may be improved by increasing leptin and decreasing resistin.

We also examined the expression of insulin receptor 2 (IR2) and adrenaline receptor 3 (ADR3), as they are most important receptors for receiving the signals for glucose metabolism [27] [28]. The black bars of Figure 6(a) show the ratios of expression observed in 3T3-BA1 cells (3-day stimulation) to those observed in 3T3-L24 cells. Unfortunately, the expression of IR2 and ADR3 observed in 3T3-BA1 cells were not enhanced.

Next, we focused on glucose transporter type 4 (GLUT4) which is the most important protein for transporting glucose into cells [29]. An increase in the level of GLUT4 is expected to promote glucose uptake, as insulin resistance was improved by the high expression of GLUT4 [30]. Line (b) of Figure 3(a) shows the expression of GLUT4 protein in the cells. Anti-GLUT4 antibody which combined with Cys3-conjugated secondary antibody was combined with GLUT4 proteins in 3T3-L24 cells and 3T3-BA1 cells (14-day stimulation). GLUT4 protein appears red under a fluorescence microscope. As shown in line (b), a small amount of GLUT4 was observed in 3T3-L24 adipocytes, but a large amount was observed in 3T3-BA1 cells. Additionally, anti-GLUT4 antibody which combined with FITC-conjugated secondary antibody was combined with GLUT4 protein, and the average fluorescence intensity in 5000 cells was determined by FACS. As shown in Figure 3(b), the average value per 3T3-BA1 cell was 2.8 times that per 3T3-L24 adipocyte. These results suggest that the glucose consumption in 3T3-BA1 beige adipocytes may be enhanced by the increase of GLUT4 protein.

We then examined the effectiveness of GLUT4 increase on the uptake rate of glucose using 2-NDGB (a fluorescent glucose derivative). 3T3-L24 and 3T3-BA1 cells cultured in 96-well plates were incubated in glucosefree DMEM (without FBS) containing 2-NDGB and in medium containing insulin. The amount of 2-NDGB in cells was proportional to the uptake amount of glucose, as the glucose analogue 2-NDGB was transported into cells instead of glucose. Figure 6(b) shows the amount of 2-NDGB in the cells. The amount of 2-NDGB in 3T3-BA1 cells was a little higher than that in 3T3-L24 cells when cultured without insulin (gray bars). When the cells were cultured with insulin, the amount of 2-NDGB contained in 3T3-BA1 cells was twice (black bars). Therefore, the uptake rate of glucose and insulin resistance in 3T3-BA1 beige adipocytes was higher than that in 3T3-L24 white adipocytes.

\section{Discussion}

Beige adipocytes are cells that resemble brown adipocytes with high fat consumption and are believed to be produced by differentiation from white adipocytes. However, few studies have found evidence supporting these 
previous findings. The purpose of this study is to confirm whether or not white adipocytes can actually differentiate to beige adipocytes and to elucidate the characteristics of beige adipocytes.

First, we searched for a compound with a high ability to induce beige adipocytes. As shown in Figure 1, we focused on the crude extracts of bofu-tsusho-san and found that kikyo extract was a strong inducer of beige adipocytes. As shown in Figure 2 and Figure 3, the 3T3-BA1 cells induced from 3T3-L24 white adipocytes by kikyo extract appeared more brownish and contained greater numbers of mitochondria than those observed in 3T3-L24 white adipocytes, and they expressed the marker genes of brown adipocytes and UCP1 protein at high levels. As these characteristics were closely similar to those of brown adipocytes, 3T3-BA1 cells were considered beige adipocytes. Therefore, our results proved that white adipocytes could indeed differentiate to beige adipocytes by stimulation with a strong inducer.

Some compounds inducing the activation of UCP1 were known. For instance, the expression of the UCP1 gene was increased by the intake of rosiglitazone, an antagonist of PPAR $\gamma$ [31], and long-term stimulation by exercise was effective in increasing the ratio of UCP1 [18]. With respect to Chinese herbal medicines, bofu-tsusho-san was effective in enhancing the secretion of adrenaline, as well as nonshivering thermogenesis, according to experiments using mice [32]-[34]. However, no effective compounds inducing the production of beige adipocytes such as kikyo extract had yet been identified. Therefore, kikyo extract might be useful as a novel anti-obese constitution drug.

Next, we elucidated the characteristics of the 3T3-BA1 beige adipocytes. As shown in Figures 3-5, the expression of the transcriptional genes of adipocytes, resistance to fatty acid, and the rates of synthesis and consumption of triglycerides in the 3T3-BA1 beige adipocytes were much higher than in 3T3-L24 white adipocytes. Additionally, as shown in Figure 3 and Figure 6, the expression of leptin genes, expression of GLUT4 protein, and uptake rate of glucose in 3T3-BA1 beige adipocytes were also much higher than in 3T3-L24 white adipocytes. These results suggested that 3T3-BA1 beige adipocytes were high-performance adipocytes that were extremely effective for metabolizing triglycerides and glucose. The characteristics of beige adipocytes were not sufficiently elucidated in previous studies, as no cell lines of beige adipocytes induced from white adipocytes had been obtained. Therefore, our results provided important information for the understanding of beige adipocytes.

Finally, we noted the stability of the beige adipocytes. When the 3T3-BA1 cells were continuously cultured in DMEM (without extract solution of kikyo), expression of PRDM16 gene was gradually decreased, and the characteristics of the beige adipocytes seemed to revert to those of white adipocytes (data not shown). These results suggested that the changes from white adipocytes to beige ones might not be a result of differentiation but simply a change to a high-performance type of cell. Several studies had suggested that beige adipocytes were a kind of white adipocyte [35] [36], findings which supported our own. However, the transgenic brown adipocytes expressing the PRDM16 gene at a high level [16] showed stable characteristics, and natural brown adipocytes taken from the body might also be stable. Therefore, we cannot deny the possibility that 3T3-BA1 beige adipocytes are different from the natural beige adipocytes present in the body, and additional expression of a gene causing permanently high expression of PRDM16 may be necessary to obtain natural beige adipocytes. Further studies are currently underway to confirm these findings.

\section{Acknowledgements}

The authors sincerely thank Ms. Mika Iwane, Yurie Seno, Yukiko Taniguchi, Ikuka Oka, Arisa Sakamoto, Chisato Niihara and Mikako Nakai for their assistance with our research. This work was supported by Grant-in-aids from the School of Human Sciences and the Research Institute at Kobe College.

\section{References}

[1] Matuzawa, Y. (2006) The Metabolic Syndrome and Adipocytokines. FEBS Letters, 580, 2917-292. http://dx.doi.org/10.1016/j.febslet.2006.04.028

[2] Chan, J.M., Rimm, E.B., Colditz, G.A., Stampfer, M.J. and Willett, W.C. (1994) Obesity, Fat Distribution, and Weight Gain as Risk Factors for Clinical Diabetes in Men. Diabetes Care, 17, 961-969. http://dx.doi.org/10.2337/diacare.17.9.961

[3] Wang, S., Moustaid-Moussa, N., Chen, L., Mo, H., Shastri, A., Su, R., Bapat, P., Kwun, I. and Shen, C.L. (2014) Novel Insights of Dietary Polyphenols and Obesity. The Journal of Nutritional Biochemistry, 25, 1-18. 
http://dx.doi.org/10.1016/j.jnutbio.2013.09.001

[4] Kim, H.M., Do, C.H. and Lee, D.H. (2010) Characterization of Taurine as Anti-Obesity Agent in C. elegans. Journal of Biomedical Science, 17, S33. http://dx.doi.org/10.1186/1423-0127-17-s1-s33

[5] Derosa, G., Maffioli, P., Ferrari, I., D’Angelo, A., Fogari, E., Palumbo, I., Randazzo, S. and Cicero, A.F. (2011) Orlistat and L-Carnitine Compared to Orlistat Alone on Insulin Resistance in Obese Diabetic Patients. Endocrine Journal, 57, 777-786. http://dx.doi.org/10.1507/endocrj.K10E-049

[6] Shiomi, N., Maeda, M. and Mimura, M. (2011) Compounds That Inhibit Triglyceride Accumulation and TNF $\alpha$ Secretion in Adipocytes. Journal of Biomedical Science and Engineering, 4, 684-691. http://dx.doi.org/10.4236/jbise.2011.411085

[7] Arechaga, I., Ledesma, A. and Rial, E. (2001) The Mitochondrial Uncoupling Protein UCP1: A Gated Pore. IUBMB Life, 52,165-173. http://dx.doi.org/10.1080/15216540152845966

[8] Kontani, Y., Wang, Y., Kimura, K., Inokuma, K.I., Saito, M., Suzuki-Miura, T., Wang, Z., Sato, Y., Mori, N. and Yamashita, H. (2005) UCP1 Deficiency Increases Susceptibility to Diet-Induced Obesity with Age. Aging Cell, 4,147-155. http://dx.doi.org/10.1111/j.1474-9726.2005.00157.X

[9] Zhou, Y., Yang, J., Huang, J., Li, T., Xu, D., Zuo, B., Hou, L., Wu, W., Zhang, L., Xia, X., Ma, Z., Ren, Z. and Xiong, Y. (2014) The Formation of Brown Adipose Tissue Induced by Transgenic Over-Expression of PPAR $\gamma 2$. Biochemical and Biophysical Research Communications, 446, 959-964. http://dx.doi.org/10.1016/j.bbrc.2014.03.033

[10] de Jesus, L.A., Carvalho, S.D., Ribeiro, M.O., Schneider, M., Kim, S.W., Harney, J.W., Larsen, P.R. and Bianco, A.C. (2001) The Type 2 Iodothyronine Deiodinase Is Essential for Adaptive Thermogenesis in Brown Adipose Tissue. The Journal of Clinical Investigation, 108, 1379-1385. http://dx.doi.org/10.1172/JCI200113803

[11] Yoneshiro, T., Aita, S., Matsushita, M., Kameya, T., Nakada, K., Kawai, Y. and Saito, M. (2011) Brown Adipose Tissue, Whole-Body Energy Expenditure, and Thermogenesis in Healthy Adult Men. Obesity (Silver Spring), 19, 13-16. http://dx.doi.org/10.1038/oby.2010.105

[12] Kozak, L.P. and Anunciado-Koza, R. (2008) UCP1: Its Involvement and Utility in Obesity. International Journal of Obesisty (Lond), 32, S32-S38. http://dx.doi.org/10.1038/ijo.2008.236

[13] Porter, C., Chondronikola, M. and Sidossis, L.S. (2015) The Therapeutic Potential of Brown Adipocytes in Humans. Frontiers in Endocrinology (Lausanne), 6, 156. http://dx.doi.org/10.3389/fendo.2015.00156

[14] Lee, Y.H., Jung, Y.S. and Choi, D. (2014) Recent Advance in Brown Adipose Physiology and Its Therapeutic Potential. Experimental \& Molecular Medicine, 46, e78. http://dx.doi.org/10.1038/emm.2013.163

[15] Seale, P., Bjork, B., Yang, W., Kajimura, S., Chin, S., Kuang, S., Scimè, A., Devarakonda, S., Conroe, H.M., Erdjument-Bromage, H., Tempst, P., Rudnicki, M.A., Beier, D.R. and Spiegelman, B.M. (2008) PRDM16 Controls a Brown Fat/Skeletal Muscle Switch. Nature, 454, 961-967. http://dx.doi.org/10.1038/nature07182

[16] Seale, P., Kajimura, S., Yang, W., Chin, S., Rohas, L.M., Uldry, M., Tavernier, G., Langin, D. and Spiegelman, B.M. (2007) Transcriptional Control of Brown Fat Determination by PRDM16. Cell Metabolism, 6, 38-54. http://dx.doi.org/10.1016/j.cmet.2007.06.001

[17] Vegiopoulos, A., Müller-Decker, K., Strzoda, D., Schmitt, I., Chichelnitskiy, E., Ostertag, A., Berriel Diaz, M., Rozman, J., Hrabe de Angelis, M., Nüsing, R.M., Meyer, C.W., Wahli, W., Klingenspor, M. and Herzig, S. (2010) Cyclooxygenase-2 Controls Energy Homeostasis in Mice by de Novo Recruitment of Brown Adipocytes. Science, 328, 1158-1161. http://dx.doi.org/10.1126/science.1186034

[18] Cypess, A.M., Chen, Y.C., Sze, C., Wang, K., English, J., Chan, O., Holman, A.R., Tal, I., Palmer, M.R., Kolodny, G.M. and Kahn, C.R. (2012) Cold but Not Sympathomimetics Activates Human Brown Adipose Tissue in vivo. Proceeding of National Academy of Science of the United States of America, 109, 10001-10005. http://dx.doi.org/10.1073/pnas.1207911109

[19] Wu, L., Zhou, L., Chen, C., Gong, J., Xu, L., Ye, J., Li, D. and Li, P. (2014) Cidea Controls Lipid Droplet Fusion and Lipid Storage in Brown and White Adipose Tissue. Science China Life Sciences, 57, 107-116. http://dx.doi.org/10.1007/s11427-013-4585-y

[20] Haas, B., Schlinkert, P., Mayer, P. and Eckstein, N. (2012) Targeting Adipose Tissue. Diabetology \& Metabolic Syndrome, 4, 43. http://dx.doi.org/10.1186/1758-5996-4-43

[21] Inokuma, K., Ogura-Okamatsu, Y., Toda, C., Kimura, K., Yamashita, H. and Saito, M. (2005) Uncoupling Protein 1 Is Necessary for Norepinephrine-Induced Glucose Utilization in Brown Adipose Tissue. Diabetes, 54, 1385-1391. http://dx.doi.org/10.2337/diabetes.54.5.1385

[22] Schulz, T.J. and Tseng, Y.H. (2013) Brown Adipose Tissue: Development, Metabolism and Beyond. Biochemical Journal, 453, 167-178. http://dx.doi.org/10.1042/BJ20130457

[23] Hondares, E., Rosell, M., Díaz-Delfín, J., Olmos, Y., Monsalve, M., Iglesias, R., Villarroya, F. and Giralt, M. (2011) 
Peroxisome Proliferator-Activated Receptor $\alpha(\mathrm{PPAR} \alpha)$ Induces PPAR $\gamma$ Coactivator $1 \alpha$ (PGC-1 $\alpha$ ) Gene Expression and Contributes to Thermogenic Activation of Brown Fat: Involvement of PRDM16. The Journal of Biological Chemistry, 286, 43112-43122. http://dx.doi.org/10.1074/jbc.M111.252775

[24] Lillioja, S., Bogardus, C., Mott, D.M., Kennedy, A.L., Knowler, W.C. and Howard, B.V. (1985) Relationship between Insulin-Mediated Glucose Disposal and Lipid Metabolism in Man. The Journal of Clinical Investigation, 75, 11061115. http://dx.doi.org/10.1172/JCI111804

[25] Yang, R. and. Barouch, L.A. (2007) Leptin Signaling and Obesity: Cardiovascular Consequences. Circulation Research, 101, 545-559. http://dx.doi.org/10.1161/CIRCRESAHA.107.156596

[26] Kusminski, C.M., McTernan, P.G. and Kumar, S. (2005) Role of Resistin in Obesity, Insulin Resistance and Type II Diabetes. Clinical Science (Lond), 109, 243-256. http://dx.doi.org/10.1042/CS20050078

[27] Lowell, B.B. and Bachman, E.S. (2003) Beta-Adrenergic Receptors, Diet-Induced Thermogenesis, and Obesity. Journal of Biological Chemistry, 278, 29385-29388. http://dx.doi.org/10.1074/jbc.R300011200

[28] Arner, P., Einarsson, K., Backman, L., Nilsell, K., Lerea, K.M. and Livingston, J.N. (1983) Studies of Liver Insulin Receptors in Non-Obese and Obese Human Subjects. Journal of Clinical Investigation, 72, 1729-1736. http://dx.doi.org/10.1172/JCI111132

[29] Mozzanega, B., Mioni, R., Granzotto, M., Chiarelli, S., Xamin, N., Zuliani, L., Sicolo, N., Marchesoni, D. and Vettor, R. (2004) Obesity Reduces the Expression of GLUT4 in the Endometrium of Normoinsulinemic Women Affected by the Polycystic Ovary Syndrome. Annals of the New York Academy of Sciences, 1034, 364-374. http://dx.doi.org/10.1196/annals.1335.038

[30] Miura, S., Kai, Y., Ono, M. and Ezaki, O. (2003) Overexpression of Peroxisome Proliferator-Activated Receptor $\gamma$ Coactivator-1 $\alpha$ Down-Regulates GLUT4 mRNA in Skeletal Muscles. Journal of Biological Chemistry, 278, 3138531390. http://dx.doi.org/10.1074/jbc.M304312200

[31] Choi, J.H., Banks, A.S., Estall, J.L., Kajimura, S., Boström, P., Laznik, D., Ruas, J.L., Chalmers, M.J., Kamenecka, T.M., Blüher, M., Griffin, P.R. and Spiegelman, B.M. (2010) Anti-Diabetic Drugs Inhibit Obesity-Linked Phosphorylation of PPAR $\gamma$ by Cdk5. Nature, 466, 451-456. http://dx.doi.org/10.1038/nature09291

[32] Yu, Q., Takahashi, T., Nomura, M., Yasuda, M., Obatake-Ikeda, K. and Kobayashi, S. (2013) Effects of Single Administered Bofutsushosan-Composed Crude Drugs on Diabetic Serum Parameters in Streptozotocin-Induced Diabetic Mice. Chinese Medicine, 4, 24-31. http://dx.doi.org/10.4236/cm.2013.41005

[33] Yoshida, T., Sakane, N., Wakabayashi, Y., Umekawa, T. and Kondo, M. (1995) Thermogenic, Anti-Obesity Effects of Bofu-Tsusho-San in MSG-Obese Mice. International Journal of Obesity and Related Metabolic Disorder, 19, 717722.

[34] Azushima, K., Tamura, K., Haku, S., Wakui, H., Kanaoka, T., Ohsawa, M., Uneda, K., Kobayashi, R., Ohki, K., Dejima, T., Maeda, A., Hashimoto, T., Oshikawa, J., Kobayashi, Y., Nomura, K., Azushima, C., Takeshita, Y., Fujino, R., Uchida, K., Shibuya, K., Ando, D., Tokita, Y., Fujikawa, T., Toya, Y. and Umemura, S. (2015) Effects of the Oriental Herbal Medicine Bofu-Tsusho-San in Obesity Hypertension: A Multicenter, Randomized, Parallel-Group Controlled Trial (ATH-D-14-01021.R2). Atherosclerosis, 240, 297-304. http://dx.doi.org/10.1016/j.atherosclerosis.2015.01.025

[35] Lehr, L., Canola, K., Léger, B. and Giacobino, J.P. (2009) Differentiation and Characterization in Primary Culture of White Adipose Tissue Brown Adipocyte-Like Cells. International Journal of Obesity, 33, 680-686. http://dx.doi.org/10.1038/ijo.2009.46

[36] Petrovic, N., Walden, T.B., Shabalina, I.G., Timmons, J.A., Cannon, B. and Nedergaard, J. (2010) Chronic Peroxisome Proliferator-Activated Receptor $\gamma(\operatorname{PPAR} \gamma)$ Activation of Epididymally Derived White Adipocyte Cultures Reveals a Population of Thermogenically Competent, UCP1-containing Adipocytes Molecularly Distinct from Classic Brown Adipocytes. Journal of Biological Chemistry, 285, 7153-7164. http://dx.doi.org/10.1074/jbc.M109.053942 